\title{
Totally Extraperitoneal (TEP) Removal of an Infected Mesh by Laparoscopy after Open Preperitoneal Repair: Initial Case Report
}

\author{
Naoto Chihara ${ }^{1}$, Keisuke Mishima ${ }^{1}$, Hideyuki Suzuki ${ }^{1}$, \\ Masanori Watanabe ${ }^{1}$, Tetsutaka Toyoda ${ }^{1}$ and Eiji Uchida ${ }^{2}$ \\ ${ }^{1}$ Institute of Gastroenterology, Nippon Medical School Musashi Kosugi Hospital, Kanagawa, Japan \\ ${ }^{2}$ Department of Surgery, Nippon Medical School, Tokyo, Japan
}

\begin{abstract}
Prosthetic mesh infection after open or laparoscopic hernia repair is a rare complication. Superficial wound infection can be resolved by treatment with a combination of antibiotics and wound drainage, whereas deep-seated mesh infection, which can lead to chronic groin sepsis, usually requires removal of the mesh. A 56-year-old Japanese man was admitted to our hospital for the treatment of deep-seated mesh infection. The patient had undergone inguinal hernia repair at another hospital 18 months earlier. The operation was prosthetic mesh repair via an anterior approach. The patient developed deep-seated mesh infection despite conservative treatment for infection, such as abscess drainage and antibiotic therapy. Since the patient eventually developed chronic groin sepsis, he was referred to our hospital, and infected mesh was removed successfully by laparoscopic surgery via a totally extraperitoneal approach. The laparoscopic approach provides several advantages, including less postoperative pain, a shorter hospital stay, and earlier rehabilitation. Furthermore, seeding of the abdominal cavity with pus never occurs with this approach unlike the laparoscopic transabdominal preperitoneal approach.
\end{abstract}

(J Nippon Med Sch 2017; 84: 45-48)

Key words: laparoscopy, mesh infection, totally extraperitoneal approach, inguinal hernia, anterior approach

\section{Introduction}

Open tension-free or laparoscopic hernia repair using a prosthetic mesh is a common standard operative procedure. The open technique involves prosthetic mesh reinforcement of the posterior wall of the inguinal canal or the preperitoneal space by the anterior approach. The laparoscopic technique can also be used to reinforce the preperitoneal space with mesh via the transabdominal preperitoneal (TAPP) or the totally extraperitoneal (TEP) approach. Deep-seated mesh infection is considered rare but once it occurs, the patient's condition can become serious. The rate of mesh infection after open prosthetic repair has been reported to vary between $0.5 \%$ and $3 \%$, whereas after laparoscopic repair, it is less than $0.16 \%^{1-8}$. Superficial wound infection usually resolves successfully within a few days or weeks with a combination of antibiotics and wound drainage. Rarely, deep-seated mesh in- fection develops a few months or a few years after mesh repair. Once deep-seated prosthetic mesh infection develops, the patient's condition can become serious with possible development of chronic groin sepsis. The symptoms include firm and painful groin swelling with red coloration of the overlying skin, and chronic groin sinus formation $^{9,10}$. Treatment with antibiotics and wound drainage is usually not effective. Therefore, most patients usually require removal of the infected mesh for resolution of the infection. In general, an infected mesh in the preperitoneal space is removed by the open procedure via a large incision. Recently, laparoscopic removal of an infected mesh has also been reported. Laparoscopic removal is associated with less pain, a shorter hospital stay and earlier rehabilitation. However, there have been few reports of laparoscopic removal of an infected prosthetic mesh via the TAPP. Herein, we report an initial case in which an

Correspondence to Naoto Chihara, Institute of Gastroenterology, Nippon Medical School Musashi Kosugi Hospital, 1-396 Kosugi-cho, Nakahara-ku, Kawasaki, Kanagawa 211-8533, Japan

E-mail: naotochihara@nms.ac.jp

Journal Website (http://www.nms.ac.jp/jnms/) 


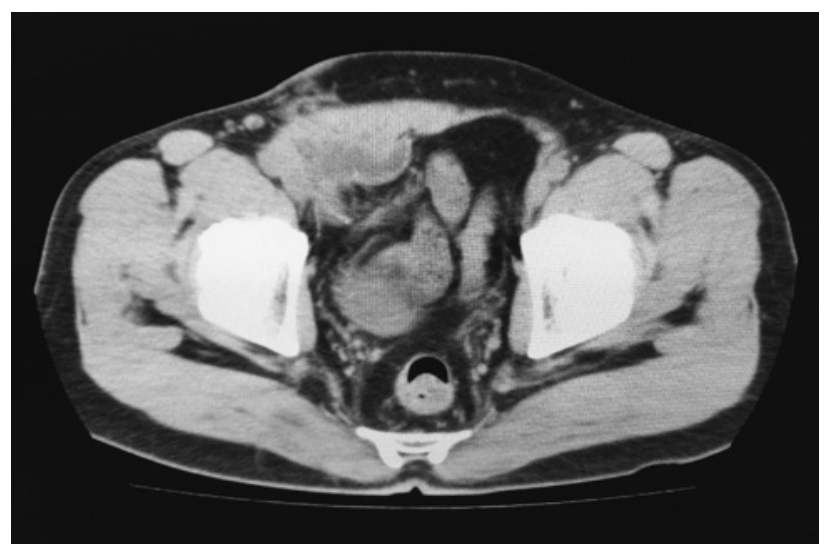

Fig. 1 Computed tomographic (CT) image showing the abscess area in the right preperitoneal space.

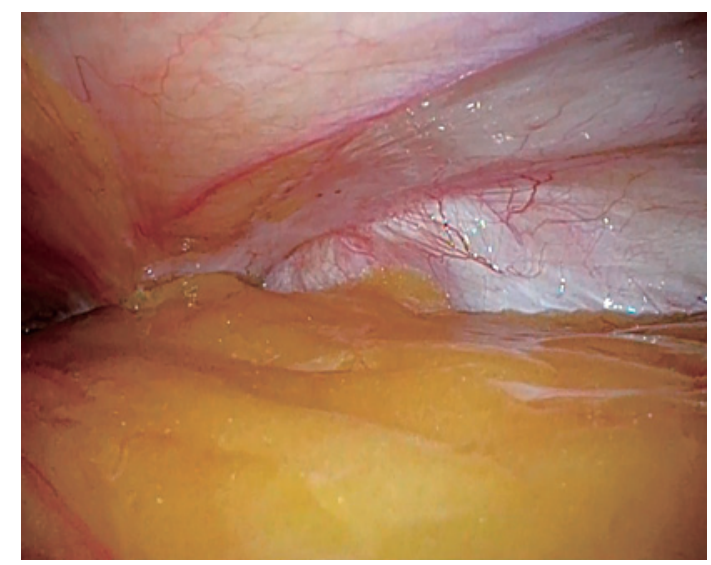

Fig. 2 Initial laparoscopic intra-abdominal view showing no adhesion formation or abscess area in the right groin.

infected mesh was successfully removed laparoscopically via the TEP.

\section{Case Report}

A 56-year-old male man was admitted to our hospital for the treatment of mesh infection that developed after inguinal hernia repair. The patient had undergone right inguinal hernia repair at another hospital 18 months earlier. The surgery was open surgery with mesh placement in the preperitoneal space via the anterior approach. The mesh consisted of a double layer of monofilament polypropylene. The patient developed deep-seated mesh infection eight months after the previous hernia repair. The infection manifested as a groin sinus with pus discharge. Abscess drainage and antibiotics therapy were performed at another hospital, but no shrinkage of the abscess space was observed. Therefore, the patient was

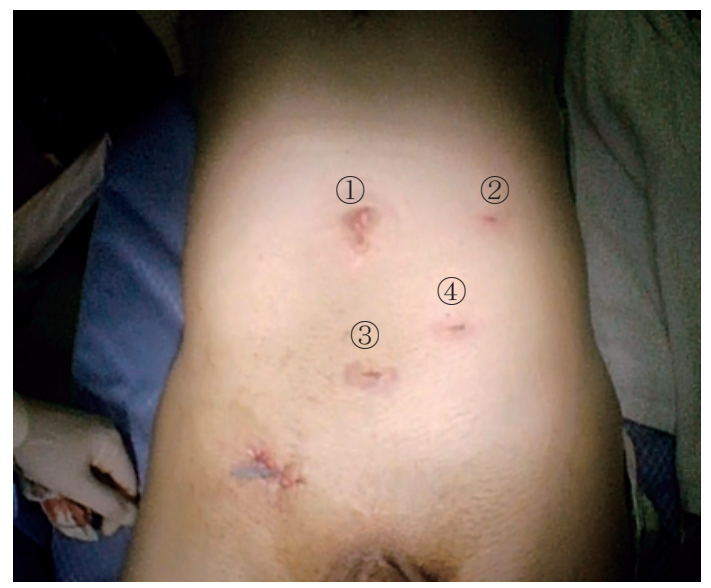

Fig. 3 Sequence of trocar placement for laparoscopic removal of an infected mesh.

referred to our hospital. Computed tomography (CT) showed abscess formation around the mesh prosthesis in the inguinal area of the abdominal wall (Fig. 1). Mesh removal was performed laparoscopically under general anesthesia via the TEP approach. The procedure is described below. First, a $5 \mathrm{~mm}$-camera trocar was inserted at umbilicus and working trocar at left lateral side. The initial laparoscopic view of the intra-abdominal space did not show any adhesion formation or abscess area in the right groin (Fig. 2). Then, the anterior fascia of the rectus on the right side was incised. An access device, consisting of a lap-protector and EZ access with a double $5 \mathrm{~mm}$ trocar, was introduced into the pre-peritoneal space. In addition, two working trocars were inserted as appropriate (Fig. 3). A strong force was needed because of the firm fibrosis around the mesh. We used an ultrasonic dissection device to separate the mesh from the abdominal wall. After some amount of dissection, the abscess area appeared (Fig. 4). The mesh was successfully removed by blunt and sharp dissection, taking care not to injure the urinary bladder, corona mortis, inferior epigastric vessels, and external iliac vessels (Fig. 5). Fortunately, there was no damage of the peritoneum. Therefore, the purulent fluid did not spread into the abdominal cavity. The mesh was extracted from the access device at the umbilicus. A suction drain was placed into the abscess cavity laparoscopically. Before finishing the operation, we confirmed the absence of injury of the urinary bladder, vas deferens, or the testicular, inferior epigastric and external iliac vessels. A sample of the purulent fluid was sent for microbiologic examination. Finally, we confirmed laparoscopically the absence of seeding of the abdominal cavity by the purulent fluid after the anterior fascia of the rectus 


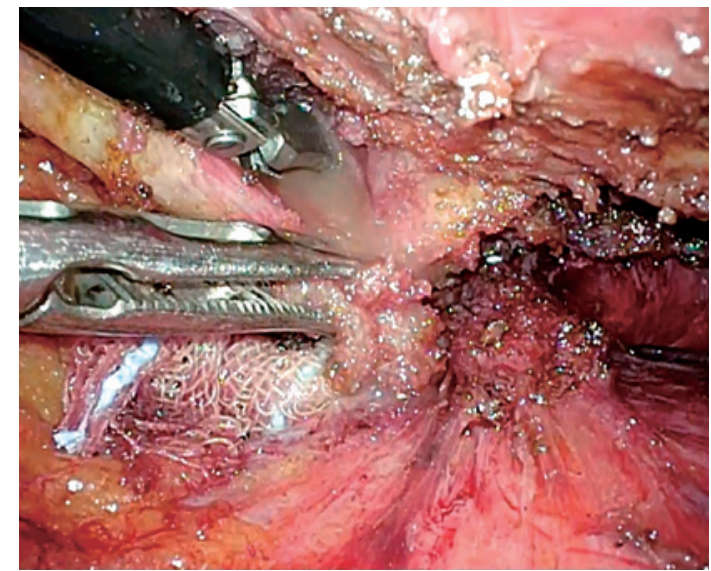

Fig. 4 Laparoscopic view showing the infected mesh with collection of pus in the preperitoneal space.

was closed. The patient had an uneventful postoperative course, except for serous discharge through the drain wound lasting for two weeks.

\section{Discussion}

In this article, we have described our experience of removal of an infected mesh after inguinal hernia repair by laparoscopy via the TEP approach. The mesh was removed by the anterior approach through a long incision. Pradeep $\mathrm{K}$ et $\mathrm{al}^{11}$ have reported laparoscopic removal of an infected mesh via the TAPP approach. However, in the laparoscopic TAPP approach, there is the risk of spread of pus into the abdominal cavity and also the risk of formation of adhesions between the peritoneum and small bowel because of the peritoneal defect in the groin. Therefore, we attempted the laparoscopic TEP approach. The laparoscopic view showed that the mesh was surrounded by firm fibrosis, as expected. Therefore, there was some difficulty in identifying and distinguishing the mesh. Initially, we dissected extraperitoneal space from the direction of the midline to identify the pubis, then following toward the exterior mesh, to avoid iatrogenic injury to the inferior epigastric vessels. Some amount of force was required to remove mesh.

It is suggested that early superficial wound infection is related to intraoperative contamination, whereas late prosthetic infection may be associated with persistent fluid collection ${ }^{12}$. Seroma is the most common postoperative complication. Pradeep et $\mathrm{al}^{11}$ suggested that repeated aspiration of seroma may cause mesh infection by the skin commensals in the early postoperative period. Then, a folded mesh after the operation may increase the probability of development of infection ${ }^{13}$. In our case, hema-

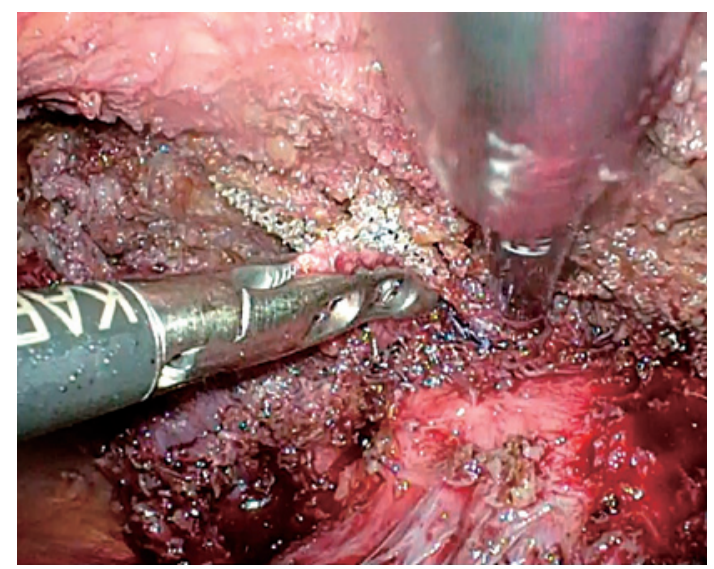

Fig. 5 Infected mesh removed by alternate blunt and sharp dissection of the surrounding tissues.

toma formation occurred after the first hernia repair. In addition, laparoscopic visualization during removal of the mesh showed that the mesh did not spread well. Thus, these factors might have led to the mesh infection in the patient. We have undertaken laparoscopic deepseated mesh hernia repairs in 450 cases so far at our hospital, without any incidence of mesh infection.

Mesh-related infection is usually caused by staphylococcus species, especially $S$. aureus, Coaglase-negative Staphylococcus, Enterococcus faecalis, Corynebacteria, Pseudomonas aeruginosa, and MRSA, although the cultures are often negative ${ }^{10,14}$. In our case, Staphylococcus epidermidis was found.

Concerning hernia recurrence after removal of the initial mesh, S. Rehman et $\mathrm{al}^{15}$ reviewed the data of 40 patients, which revealed that the hernia recurrence rate after removal of the mesh was less than $5 \%$. Taylor et $\mathrm{al}^{9}$ reported that only two of their patients developed recurrent hernia: one of them was asymptomatic and is currently under observation, while the other required opentension free hernioplasty for a right side recurrence after previous bilateral laparoscopic hernia repair. Although this operation took place two years after the mesh removal, the patient developed a further episode of chronic groin sepsis, which again necessitated removal of the mesh to obtain resolution of the symptoms ${ }^{9}$. Hernia recurrence may be uncommon following mesh removal, suggesting that the strength of the mesh lies in the fibrous reaction evoked within the transversalis fascia by the prosthetic material ${ }^{16}$. However, these case series are retrospective studies with relatively small sample sizes and insufficient follow-up periods after removal of the mesh. It is noteworthy that Pradeep $\mathrm{K}$ et $\mathrm{al}^{11}$ reported a 
recurrence rate of hernia following laparoscopic removal of the mesh after hernia repair of $20 \%$. Therefore, our case needs careful follow-up for timely detection of any evidence of recurrence.

\section{Conclusion}

Laparoscopic removal of an infected mesh by the TEP approach has several advantages, such as less postoperative pain, a shorter hospital stay and earlier rehabilitation. Furthermore, seeding of the abdominal cavity by pus never occurs with this approach as compared to the laparoscopic TAPP approach.

Conflict of Interest: The authors declare that they have no conflict of interest.

\section{References}

1. Birth M, Friedman RL, Mellullis M, Weiser HF: Laparoscopic ransabdomina preperitoneal hernioplasty: results of 1000 consecutive cases. J Laparoendosc Surg 1996; 6: 293-300.

2. Bittner R, Leibl B, Kraft K, Daubler P, Schwarz J: Laparoscopic hernioplasty (TAPP). Zentralbl Chir 1996; 11: 313319.

3. Hernandez-Richter T, Meyer G, Schardey HM, Rau HG, Schildberg FW: Transabdominal preperitoneal hernia repair (TAPP). Zentralbl Chir 1999; 124: 657-663 (German).

4. Kapiris SA, Brough WA, Royston CM, O'Boyle C, Sedman PC: Laparoscopic transabdominal preperitoneal (TAPP) hernia repair: a 7-year two-center experience in 3017 patients. Surge Endosc 2001; 15: 972-975.

5. Litwin DE, Pham QN, Oleniuk FH, Kluftinger AM, Rossi L: Laparoscopic groin hernia surgery: the TAPP procedure. Transabdominal preperitoneal hernia repair. Cam J Surg 1997; 40: 192-198.
6. Schmedt C, Daubler P, Leibll BJ, Kraft K, Bittner R: Simultaneous bilateral laparoscopic inguinal hernia repair: an analysis of 1336 consecutive cases at a single center. Surg Endosc 2002; 16: 240-244.

7. Schultz C, Baca I, Gotozen V: Laparoscopic inguinal hernia repair. Surg Endosc 2001; 15: 582-584.

8. Tamme C, Scheidbach H, Hampe C, Schneider C, Kockerling F: Totally extraperitoneal endoscopic inguinal hernia repair (TEP). Surg Endosc 2003; 17: 190-195.

9. Taylor SG, O'Dwyer PJ: Chronic groin sepsis following tension-free hernioplasty. Br J Surg 1999; 86: 562-565.

10. Delikoukos S, Tzovaras G, Liakou P: Late-onset deep mesh infection after inguinal hernia repair. Hernia 2007; 11: $15-17$.

11. Pradeep K, Chowbey PK, Khullar R, Shama A: Laparoscopic management of infected mesh after laparoscopic inguinal hernia repair. Surg Laparosc Endosc Percutan Tech 2015; 25: 125-128.

12. Mann DV, Prout J, Havranek E, Gould S, Darzi A: Lateonset deep prosthetic infection following mesh repair of inguinal hernia. Am J Surg 1998; 176: 12-14.

13. Foschi D, Corsi F, Cellerino P, Trabucchi A, Trabucci E: Late rejection of the mesh after laparoscopic hernia repair. Surg Endosc 1998; 12: 455-457.

14. Stremizer S, Bachleitner-Hofmann T, Gradl B: Mesh Graft Infection Following Abdominal Hernia Repair; Risk Factor Evaluation and Strategies of Mesh Graft Preservation. A Retrospective Analysis of 476 Operations. World J Surg 2010; 34: 1702-1709.

15. Rehman S, Khan S, Pervaiz A, Perry EP: Recurrence of inguinal herniae following removal of infected prosthetic meshes: a review of the literature. Hernia 2012; 16: 123126.

16. Fawole AS, Chaparala RP, Ambrose NS: Fate of the inguinal hernia following removal of infected prosthetic mesh. Hernia 2006; 10: 58-61.

(Received, January 17, 2016)

(Accepted, October 13, 2016) 